\title{
Saberes e Práticas de uma Equipe Multiprofissional: atendimento à crise no Centro de Atenção Psicossocial
}

\author{
José Adelmo da Silva Filho ${ }^{l}$; Vanessa de Morais Braga ${ }^{2}$; Maria do Livramento Alencar de Holanda ${ }^{3}$
}

\begin{abstract}
Resumo: O objetivo deste estudo é analisar a condução dos usuários em crise no Centro de Atenção Psicossocial Geral de Iguatu, na perspectiva dos profissionais do serviço. Método: pesquisa qualitativa, os dados foram coletados através de entrevista semiestruturadas nos meses de janeiro e fevereiro 2016, aplicada a todos os profissionais de saúde mental que atuam diretamente com pacientes em sofrimento, composta por: médico psiquiatra, residentes de psiquiatria, enfermagem, técnicos de enfermagem, assistente social, psicólogo, pedagogo, recepcionista e vigia. A análise e interpretação dos dados foram realizados de acordo com a técnica de Análise de Conteúdo de Minayo. Resultados e Discussão: como resultado percebeu-se que o dimensionamento do tratamento em situação de crise, atualmente, está bastante associado a terapêutica estabelecida entre equipe de saúde, usuário e família, bem como a manutenção de vínculos e a singularidade dos sujeitos, embora haja fragilidades no tocante a estrutura física do serviço, ao recursos humanos e capacitação dos profissionais. Conclusão: propõe-se a efetivação de novas condições de trabalho e ações voltadas a educação dos familiares, uma vez que é necessário a corresponsabilização no cuidado ao usuário.
\end{abstract}

Descritores: Saúde Mental. Intervenção na Crise. Cuidados de Assistência à Saúde.

\section{Knowledge and Practices of a Multiprofessional Team: Processing the crisis in the Psychosocial Care Center}

\begin{abstract}
The objective was to analyze the conduct of users in crisis in the General Psychosocial Care Center of Iguatu, from the perspective of service professionals. Qualitative research method was used and the data were collected through a semistructured interview in January and February 2016, applied to all mental health professionals who work directly with suffering patients, consisting of: psychiatrist, residents of psychiatry, nursing, technicians nurse, social worker, psychologist, pedagogue, receptionist and watchman. Data analysis and interpretation were performed according to the Minayo Content Analysis technique. As a result, it was observed that the dimensioning of the treatment in a crisis situation, is nowadays very associated with established therapy among the health team, user and family, as well as the maintenance of the bonds and the singularity of the subjects, although there is weaknesses in the physical structure of the service, human resources and professional training. It can be concluded that a proposal to implement new working conditions and actions aimed at the education of family members, since it is necessary to co-responsibility in the care of the user.
\end{abstract}

Keywords: Mental Health. Intervention in the Crisis. Health Care Care.

\footnotetext{
${ }^{1}$ Enfermeiro. Especialista em Saúde Mental. Residente em Saúde Mental Coletiva pela ESP-CE. Iguatu-CE, E-mail: adelmof12@gmail.com

${ }^{2}$ Assistente Social. Especialista em Políticas Públicas e Gestão do SUAS pela Faculdade Vale do Salgado; Especialista na modalidade de residência em Saúde Mental Coletiva pela Escola de Saúde Pública do Ceará. E-mail: vanessamb@outlook.com

${ }^{3}$ Assistente Social. Especialista em Administração de Empresas pela Faculdade Leão Sampaio. Mestra em Ensino na Saúde pela Universidade Estadual do Ceará. E-mail: liliholanda@uol.com.br
} 


\section{Introdução}

Sabe-se que a maior parte dos pacientes psiquiátricos são internados em momentos de crise. A crise pode se manifestar em vários momentos do desenvolvimento humano, diante de situações de perdas, ameaças e estresses, alterando o equilíbrio normal do indivíduo (SILVA; DIMENSTEIN, 2014).

Conforme dispõe Foucault (1979), a crise no seu sentido tradicional era entendida como uma demanda instável, contraditória e imprevisível que deve ser entendido pela sociedade como algo que deve ser normatizado. Tal relação entre a noção de crise e o significado de adequação social é destacada por Silva (2007), quando este discute que o sujeito que apresenta transtornos mentais é o inadequado, ou seja, aquele que rompe com a 'doutrina dos costumes'.

Contudo, Luzio (2010) vem romper com esta ideia restrita da crise quando afirma que a crise produz experiências dolorosas, marcadas pela solidão, incerteza, temor e estranhamento. Nesse contexto, a crise seria compreendida como um momento específico do indivíduo no qual "efervescem questões, afetos, gestos e comportamentos variáveis singulares, que afetam em graus diversos a vida cotidiana da própria pessoa e daqueles de seu convívio" (COSTA, 2007).

A situação de crise é caracterizada como uma condição em que há um distúrbio do pensamento, emocional ou comportamental, no qual é necessário e de fundamental importância a realização de um atendimento adequado e célere sempre focado no paciente, de forma que se evitem maiores prejuízos físicos, emocionais e até mesmo cessar os possíveis riscos a sua vida ou a de outros (LIMA et al., 2012).

O processo de crise do usuário é caracterizado como um momento em que o sofrimento é muito intenso que gera consequências não somente no indivíduo, como também na sua família. Dessa forma, ver-se a necessidade da intervenção imediata de uma equipe multiprofissional, com o intuito de evitar maiores danos à própria pessoa ou a terceiros (SILVA; SILVA; OLIVEIRA, 2012).

Atualmente, pela Política de Saúde Mental, o local que deve atender as crises é o Centro de Atenção Psicossocial - CAPS. Assim, o serviço deve dispor de uma equipe qualificada, preparada e sensibilizada aos princípios da Política de Humanização, preparados para manejar as situações de agitação e agressividade intensa. 
Diante desse contexto, surgem as seguintes questões: como a equipe multiprofissional atua no atendimento à crise no CAPS? Qual a visão que estes profissionais apresentam sobre a reforma psiquiátrica? Qual o perfil dos usuários em crise?

A investigação apresenta como objetivo analisar a condução dos usuários em crise no Centro de Atenção Psicossocial Geral (CAPS III) de Iguatu, sob o olhar dos profissionais do serviço.

\section{Materiais e Métodos}

Tendo em vista que o tema desta pesquisa se centra no atendimento em situações de crise a partir da percepção da equipe multiprofissional, justifica-se a escolha do tipo de pesquisa de acordo com a abordagem qualitativa.

Para realização da pesquisa foi escolhido o município de Iguatu, mais especificamente o Centro de Atenção Psicossocial Geral (CAPS III), o primeiro implantado no nordeste, no ano de 1991, resultado da Luta Antimanicomial encabeçada pelos médicos do município.

No que se refere aos leitos destinados no serviço para observação em situações de crises, na coleta dos dados, dividia-se em duas enfermarias sendo uma com cinco leitos masculinos e outra com dois leitos femininos.

Diante do exposto, foram elencados todos os profissionais de saúde mental que acolhem os pacientes em sofrimento, composta por: médico psiquiatra, enfermeiro, técnico de enfermagem, assistente social, psicólogo, pedagogo, vigia e recepcionista. Sendo respeitados os princípios éticos de participação voluntária, tendo como critério de inclusão ser profissional da saúde que preste cuidados aos pacientes psiquiátricos, e que trabalham na instituição a mais de 6 meses.

Os dados foram coletados através de entrevista semiestruturada a onze profissionais do CAPS III, nos meses de janeiro e fevereiro de 2016, sendo gravada para posterior descrição e análise dos dados, que por sua vez, foram ponderados de acordo com a Análise de Conteúdo de Minayo. Na análise dos dados, após leitura e releitura do material coletado, através de entrevistas e observação do campo, emergiram três grandes categorias temáticas: 1) crise e

864 Id on Line Rev. Mult. Psic. V.12, N. 42, p. 862-874, 2018 - ISSN 1981-1179 Edição eletrônica em http://idonline.emnuvens.com.br/id 
critérios para classificação; 2) perfil dos usuários em crise; 3 ) atenção à crise: o tratamento ideal na visão do profissional de saúde mental.

A presente pesquisa obteve aprovação pelo Comitê de Ética em Pesquisa com Seres Humanos da Escola de Saúde Pública do Ceará, sob parecer $\mathrm{n}^{\circ}$ 1.374.360, CAAE 51103015.7 .0000 .5037 .

\section{Resultados e Discussão}

\section{Crise e critérios para classificação}

A palavra crise é imbricada de elementos que trazem um amplo sentido de mudança transitória. Assim como sua definição pela origem grega (krisis) revela um estado de tomada de decisão (ÁVILA; BERLINCK, 2014).

Ao longo da história o termo passou a assumir um caráter de negatividade, uma vez que, em 1944, Erich Lindernann's, fundamentou a "teoria da crise" que abordava as consequências psicológicas causadas nos seres humanos após acontecimentos fatais ou catástrofes, ou seja, uma concepção que atribui o adoecer psíquico quase que inteiramente a causas externas ou sociais (ORNELAS, 1997).

Após anos de retrocesso, encarceramentos e medicalização da loucura, vivencia-se um processo de mudança assistencial na saúde mental. Com o advento da Reforma Psiquiátrica, iniciada no fim da década de 1970, e fortalecida pelo Movimento de Luta Antimanicomial de 1987, pode-se perceber a mudança de paradigmas e mentalidades, que defendem ainda hoje uma sociedade sem manicômios e o cuidado em liberdade (BARBOSA; COSTA; MORENO, 2012).

Como iniciou-se a discussão, a palavra crise pode remeter a vários aspectos. Contudo, especificar crise no campo da saúde mental exige considerar várias teorias já criadas e as visões particulares dos indivíduos, que no caso deste estudo, centram-se nos profissionais de um Centro de Atenção Psicossocial Geral.

Na percepção destes profissionais, pode-se dizer que: 
“Crise é quando o paciente está num estado de agitação, alucinando, com delírios, e está precisando de um acompanhamento com urgência do psiquiatra para ser avaliado” (Entrevistado 2)

Como também a crise pode ser vista quando "o usuário não está na capacidade completa de gerir a sua vida, ou de gerir a sua vida de uma maneira saudável" (Entrevistado 11)

Através dos depoimentos percebe-se que há, entre os profissionais investigados, concordância quanto à definição de crise. Observa-se que os modos de reconhecimento da crise derivam das definições semelhantes, embora particularizadas em cada prática profissional.

Sendo assim, ao analisar todas as respostas pode-se fundamentar que, atualmente, o processo da crise é entendido como um processo de agudização do transtorno mental, um momento de maior intensidade, de mudanças no comportamento, em que o usuário necessita ser assistido mais de perto e com maior agilidade.

No tocante aos critérios estabelecidos pelo serviço no momento de identificação das situações de crises, embora os profissionais afirmarem que não existem protocolos definidos pela equipe e coordenação, o usuário é atendido, primeiramente, na recepção, que por sua vez o encaminha para o acolhimento com um profissional de nível superior responsável pelas triagens e reavaliações, e caso necessário para atendimento com o médico psiquiatra.

Na atenção à crise é de fundamental importância algumas recomendações úteis para a abordagem e intervenção. Considera-se que é imprescindível a observação minuciosa da cena de urgência para a articulação de estratégias e estimativa dos riscos ali presentes.

\begin{abstract}
A avaliação da cena da crise, que consiste na leitura das diversas dimensões que atuam no seu contexto concreto de origem e sustentação, é o elemento básico e inaugural da avaliação de Risco e Vulnerabilidade. Ela envolve a detecção dos elementos de risco ambientais e materiais, comuns a toda avaliação da cena no campo das urgências, os quais são muito relevantes nas abordagens domiciliares e de rua, sejam aquelas realizadas pelo SAMU, Equipes de Saúde da Família ou da Saúde Mental. A avaliação da cena deve ser resgatada no ambiente de transferência de cuidado entre equipes de abordagem in loco e aquelas que farão a continuidade da assistência, por exemplo, na interface entre SAMU e CAPS. ${ }^{(11)}$
\end{abstract}

Durante a crise, não se deve ignorar a urgência, a pressa e os elementos objetivos que nos assinalam a necessidade da tomada de decisões. Porém, a estratégia principal deve ser criar as condições de possibilidades para que a fala, a palavra, as histórias, os sentidos possam destacar o sujeito da crise (ÁVILA; BERLINCK, 2014). 
As intervenções devem deixar lugar para que a crise seja ressignificada e superada. Conseguir identificar qual a emoção ou afeto está oculto na situação de crise, é uma importante ferramenta para um manejo adequado.

Dessa forma, após a realização do acolhimento inicial:

"No CAPS Geral de Iguatu, são usados três critérios para se estabelecer se é urgência ou não, que é a agitação psicomotora, a presença de delírios e alucinações e risco, que a gente chama de risco a suicídio, mas que a gente tem que contar também como risco a outras pessoas" (Entrevistada 9)

Para tal avaliação, é realizado o atendimento individual, para analisar as subjetividades e contextos internos e externos do indivíduo, bem como é utilizado pelo serviço um instrumental que apresenta alguns quesitos objetivos onde é possível identificar o grau da urgência daquele atendimento. Contudo, os profissionais do serviço ainda não se adaptaram ao instrumental, como relatado a seguir:

"[...] Mas agora com o reajustamento do serviço a gente está até parando de usar a ficha, porque ela além de ser muita complexa, ela pergunta coisa demais que em uma situação de crise, fica até dificil de você fazer o preenchimento [...]" (Entrevistado 9)

Assim, diante das exposições e corroborando com alguns autores, pode-se observar que alguns princípios orientam as intervenções e são de extrema validade, como:

\footnotetext{
- estar atento à postura e à missão, mantendo-se sempre focado no objetivo. Procurar ser claro, usar frases curtas e objetivas. Escutar, permitir tempo e espaço para a fala do usuário e dos familiares, procurando delimitar o problema e conflito central a partir dos enunciados;

- nunca enveredar por situações nas quais o usuário, já ameaçado, sinta-se mais acuado. Não colocar alternativas do tipo tudo ou nada, ganhar ou perder. Tentar alinhar os seus objetivos com o do usuário

- saber intervir na rede familiar e social, não restringir as intervenções ao usuário em crise. Atentar para pessoas e familiares que são referência e têm a confiança do usuário e solicitar, com tato e gentileza, a saída de cena de um familiar que seja fonte de conflito e tensão (ZEFERINO, 2015).
}

\section{Perfil dos usuários em crise}

Considerando os indicadores de situação de saúde, gênero, faixa etária e condição de acompanhamento, pode-se concluir que, de acordo com a fala e percepção dos profissionais do serviço, os usuários atendidos em situação de crise no CAPS Geral, são na sua maioria 
mulheres, numa estimativa de 55\%; do total de onze entrevistados, seis responderam mulheres, dois responderam homens, e três afirmaram que a demanda é balanceada no tocante ao gênero.

Com relação à variável da idade, a faixa etária de maior incidência está entre 30 a 40 anos; do total de onze entrevistados, seis responderam essa faixa etária.

E no tocante a presença de acompanhante no atendimento em situação de crise, a maioria respondeu que os usuários apresentam um familiar ou amigo que o acompanhe durante os procedimentos. Contudo, em boa parte das falas dos entrevistados é exposta a dificuldade de se trabalhar com a família, uma vez que são enfrentados inúmeros desafios, como é possível observar a seguir:

"Porque no internamento muitas vezes precisa de um responsável para encaminhar, e aqui na observação no CAPS precisa de um familiar e muitas vezes a gente não tem, as famílias muitas vezes não tem esse envolvimento" (Entrevistado 8)

“Normalmente a família foge quando se está pensando em alguma internação” (Entrevistado 11)

Na tentativa de superação das condições sociais atuais e no acesso ao serviço de saúde de qualidade, a informação é entendida como sendo de fundamental importância para o conhecimento da realidade e inovação na situação de saúde.

Com a pesquisa, foi possível confirmar a fragilidade da saúde mental quanto a quantificação de indicadores. Percebe-se a necessidade da construção de salas de situação, como uma ferramenta capaz de realizar um diagnóstico situacional através da análise de dados e informações de saúde, permitindo uma nova visão sobre a realidade, servindo para subsidiar decisões.

Com os indicadores é possível identificar que ainda não existe na Saúde Mental um sistema de controle de dados que seja fidedigno a prática realizada, assim como confirma este profissional em sua fala:

"Eu não consigo traçar um perfil exato, porque nem sou só eu que faço esses atendimentos, porque se fosse seria mais fácil. Esse é o tipo de atendimento que não é registrado [...]. A gente sabe que há fragilidade dos registros sim, isso deveria está muito bem estabelecido e registrado, mas não está" (Entrevistado 9)

Dessa forma, é possível concluir que a deficiência de registros das ações realizadas compromete o acompanhamento sistemático, sendo assim a sala de situação é considerada uma 
estratégia possível identificar os números reais e avaliar se as intervenções estão obtendo resultados relevantes.

\section{Atenção à crise: o tratamento ideal na visão do profissional da saúde mental}

Para iniciar tratamento no CAPS, atualmente, ao usuário não é exigido referência de outro serviço, podendo assim, chegar por demanda espontânea. No entanto, vários usuários ainda chegam encaminhados pela Estratégia de Saúde da Família (ESF), com referência apontando o motivo da demanda e os sintomas apresentados.

Em situações de crise, foi constatado nas respostas dos profissionais que os pacientes chegam de duas formas, por demanda espontânea acompanhados pela família ou pelos serviços de urgência e emergência, como confirmam as falas:

"Varia muito, vem pela atenção básica, vem pelo hospital, pela UPA, por demanda espontânea, mas agora a maioria é demanda espontânea. Tipo a família ver que o paciente está em crise e trazem logo pro atendimento" (Entrevistado 2)

“A gente sempre orienta as pessoas que não é obrigatório encaminhamento para a pessoa procurar o CAPS. No caso da pessoa em crise, geralmente eles vem encaminhados do hospital regional, SAMU, e alguns casos, por não ser a primeira, vez, a família já sabe como é o procedimento e traz direto pra cá" (Entrevistado 4)

É possível observar que o cuidado ao ser humano é um ato complexo, tornando-se necessário muito conhecimento, empatia, vínculo e sensibilidade. O cuidado envolve também intervenções como escutar, tocar, sentir, ajudar nas atividades que o usuário apresenta dificuldade. Os profissionais do serviço, durante os atendimentos e acolhimentos, devem oferecer segurança para a realização da conversa, objetivando a princípio, minimizar ou até mesmo aliviar a ansiedade, os medos e discutir situações vivenciadas por aquele indivíduo.

A clínica realizada deve ser considerada para além das expressões que a doença traz. Precisa-se de uma noção de clínica que seja ampliada em conceitos. A ideia de clinica ampliada pressupõe que haja um compromisso enfático com o sujeito em adoecimento, em sua singularidade; deve-se assumir uma responsabilidade sobre os usuários do serviço, buscando sempre o exercício da intersetorialidade, bem como reconhecer os limites dos conhecimentos e 
tecnologias, mas nunca deixando de mão o compromisso ético da profissão e para com o sujeito (BRASIL, 2008).

Espera-se também como estratégia de interferência no processo de saúde, um atendimento humanizado, que garanta a atenção integral e resolutiva ao usuário. Que valorize os diferentes sujeitos, corroborando para o exercício da autonomia e protagonismo destes.

De acordo com a Política Humaniza SUS, o sistema deve ser contagiado por esta atitude humanizadora, articulando-se através deste eixo. Trata-se, sobretudo, de destacar o aspecto subjetivo presente em qualquer ação humana, em qualquer prática de saúde (MARTINS; LUZIO, 2017).

Pode-se dizer que a rede de humanização em saúde é uma rede de construção permanente de laços de cidadania, de um modo de olhar cada sujeito em sua especificidade, sua história de vida, mas também de olhá-lo como sujeito de um coletivo, sujeito da história de muitas vidas.

Ainda tomando como base a Política Nacional de Humanização, a humanização é um pacto, um processo coletivo que só pode acontecer a partir da construção e troca de saberes, através do trabalho em rede com equipes multiprofissionais, da identificação das necessidades, desejos e interesses desenvolvidos, do reconhecimento de gestores, trabalhadores e usuários como sujeitos ativos e protagonistas das ações de saúde, e da criação de redes solidárias e interativas, participativas e protagonistas do SUS (BRASIL, 2010).

Considerando esse pressuposto, e analisando os depoimentos dos profissionais do CAPS, ver-se que a equipe entende por cuidado em saúde e tratamento ideal no tocante a crise, que é o foco deste estudo, a necessidade de "apoio familiar, depois estruturas favoráveis do serviço e uma equipe comprometida" (Entrevistado 6). Como também prevê a necessidade:

"Passar pro triagem, ter o acompanhamento médico, a gente fazer um plano terapêutico com a família, se for possível estabilizar e fazer o tratamento em casa, a gente acertar isso ai com eles e deixar bem claro, bem definido, o que a gente vai fazer pra tratar o paciente, o que a gente vai ter como objetivo no tratamento" (Entrevistado 8)

Mais da metade das respostas pondera que para realização do tratamento ideal é de fundamental importância as intervenções interdisciplinares. Tendo em vista que no CAPS o atendimento deve ser de ordem psicossocial e necessita garantir a articulação e integração dos 
pontos de atenção das redes de saúde no território, qualificando o cuidado por meio do acolhimento, do acompanhamento contínuo e da atenção às urgências.

Contudo, percebe-se que ainda há fragilidades no tocante ao trabalho em equipe. Os profissionais consideram que:

"O ideal mesmo seria o atendimento multiprofissional, mas não está sendo realizado na sua totalidade, até pela questão do serviço mesmo, ainda está muito deficiente” (Entrevistado 3)

"Pensando nas políticas e no CAPS como psicossocial acaba que os tratamentos mesmo sendo dentro do CAPS, numa pauta de reforma psiquiátrica, de reforma da saúde mental no Brasil, acaba que o tratamento dado aos pacientes ainda é muito médico, falta um tanto de multidisciplinar" (Entrevistado 11)

Ao observar também a prática desses profissionais, foi possível confirmar a deficiência na efetivação do trabalho em equipe. Uma vez que a demanda do serviço é muito grande, atendendo a população de nove municípios referenciados pela $18^{\mathrm{a}} \mathrm{CRES}$, os recursos humanos são reduzidos, e a própria estrutura física não favorece o atendimento integral. Assim como dispõe um dos sujeitos da pesquisa:

"[...] Atendimento ideal é quando um paciente que está em crise chegar no serviço para ser atendido, deva ter vários olhares sobre aquelas pessoas, deva ter uma equipe capacitada, nós não temos. [...] Por exemplo, uma pessoa que vai ser internada tem que ser feito o PTS ali, junto com a pessoa, com a família [...] (Entrevistado 7)

No entanto, há potencialidades no serviço que demonstram que a equipe é detentora e defensora dos princípios da Reforma Psiquiátrica e do Movimento da Luta Antimanicomial, como observado na coleta dos dados pela pesquisadora.

Atualmente, as estratégias de intervenções têm a seguinte compreensão:

\footnotetext{
"Intervenção ideal para mim realmente tem que ser no CAPS. Tem que ser iniciada pela escuta, não só do familiar, mas também do paciente. Por que essa crise pode sim ser gerada por algo, ou falta de medicamento, mas também há um fator externo de alguma situação que desestabilizou o paciente. [...] Mas definitivamente, as intervenções devem ser feitas no CAPS, e em caso de algumas situações em que tem agitação psicomotora ou que induzam o paciente ter um comportamento de risco, eu acho que tem que ser feita no serviço com permanência 24 h durante alguns dias, até pra gente garantir que esse paciente faça uso da medicação e não coloque sua própria vida em risco." (Entrevistado 9)
}

Embora, algumas intervenções ainda permanecem, como por exemplo, a dificuldade do acompanhamento para prevenir e reduzir os episódios de crise. Seja pela não administração, ou 
pela administração das medicações em horários errados, realizada pelos familiares aos usuários; seja pela ideia do atendimento centrado no médico e a fragilidade do trabalho em equipe.

\section{Considerações Finais}

Como pode-se verificar, o dimensionamento do tratamento em situação de crise, atualmente, está bastante associado a terapêutica estabelecida entre equipe de saúde, usuário e família, bem como a manutenção de vínculos e a singularidade dos sujeitos.

Sabe-se que o CAPS não é o incumbido de dar conta de tudo. Ele, em si, não é substitutivo do hospital psiquiátrico no sentido de vir a ocupar o lugar do hospital, é uma rede diversificada de serviços e dispositivos territoriais que possam dar conta da complexidade do sofrimento psíquico, estimulando e potencializando os recursos existentes na comunidade.

Através dos depoimentos dos profissionais que trabalham no CAPS Geral de Iguatu, pode-se concluir que o CAPS precisa investir mais no seu potencial para o atendimento de crises e quadros agudos, introduzindo tecnologias norteadas pelos princípios da clínica ampliada, principalmente, no que se refere ao trabalho em equipe, que possibilitem ao usuário e sua família, maiores retornos e suporte nesses momentos de intenso sofrimento.

Em outras palavras, o que se propõe é a efetivação de novas condições de trabalho. Uma vez que foi frisado na maioria das falas, frágil estrutura física do serviço e falta de recursos humanos para atuar no acolhimento e acompanhamento destes usuários. Ressalta-se também, a grande importância de um trabalho educativo às famílias destes sujeitos, uma vez que é necessário a corresponsabilização durante o tratamento.

\section{Referências}

ÁVILA, C.S.; BERLINCK, M.T. Reflexões sobre crise e estabilização em Psicopatologia Fundamental. Tempo psicanal. [Internet]. v. 46, n. 2, p. 270-286. 2014. Disponível em: http://pepsic.bvsalud.org/scielo.php?script=sci_arttext\&pid=S010148382014000200006\&lng $=\mathrm{pt}$ 
BARBOSA, G.; COSTA, T.; MORENO, V. Movimento da luta antimanicomial: trajetória, avanços e desafios/ The anti-asylum fight movement: trajectory, progress and challenges. Cadernos Brasileiros de Saúde Mental/Brazilian Journal of Mental Health, v. 4, n. 8, p. 45-50. 2012. Disponível em: http://incubadora.periodicos.ufsc.br/index.php/cbsm/article/view/2017

BRASIL. Ministério da Saúde. Secretaria de Atenção à Saúde. Núcleo Técnico da Política Nacional de Humanização. Clínica ampliada, equipe de referência e projeto terapêutico singular / Ministério da Saúde, Secretaria de Atenção à Saúde, Núcleo Técnicoda Política Nacional de Humanização - 2. ed. - Brasília: Ministério da Saúde, 2008.

BRASIL. Ministério da Saúde. Secretaria de Atenção à Saúde. Núcleo Técnico da Política Nacional de Humanização. Humaniza SUS: Documento Base para Gestores e Trabalhadores do SUS/Ministério da Saúde, Secretaria de Atenção à Saúde, Núcleo Técnico da Política Nacional de Humanização. - $4^{\mathrm{a}}$ ed. $4^{\mathrm{a}}$ reimp. - Brasília: Ed. Ministério da Saúde, 2010.

COSTA, M.S. Construções em torno da crise. Saberes e práticas na atenção em saúde mental e produção de subjetividades. Arquivos Brasileiros de Psicologia, Rio de Janeiro, v. 59, n. 1, p. 94-108. 2007.

Disponível em: http://pepsic.bvsalud.org/scielo.php?script=sci_arttext\&pid=S1809-52672007000100010

FOUCAULT M. A casa dos loucos. In: . Microfísica do poder. Rio de Janeiro: Edições Graal. p. 113-128. 1979.

LIMA, M. et al. Signs, meanings and practices of crisis management in psychosocial care centers. Interface - Comunic., Saude, Educ., v. 16, n.41, p. 423-34. 2012. DOI: http://dx.doi.org/10.1590/S1414-32832012000200011

LUZIO, C.A. Atenção psicossocial: reflexões sobre o cuidado em saúde mental no Brasil. 2010. Tese (Livre-Docência) - Faculdade de Ciências e Letras de Assis, Universidade Estadual Paulista, Assis, 2010.

MARTINS, C.P.; LUZIO, C.A. Política HumanizaSUS: ancorar um navio no espaço. Interface (Botucatu) [Internet]. v. 21, n. 60, p. 13-22. 2017. DOI: http://dx.doi.org/10.1590/180757622015.0614

ORNELAS, J. Psicologia comunitária: Origens, fundamentos e áreas de intervenção. Aná. Psicológica, Lisboa, v. 15, n. 3, p. 375-388. 1997. Disponível em: http://www.scielo.mec.pt/scielo.php?script=sci_arttext\&pid=S0870-

$82311997000300002 \& \operatorname{lng}=$ pt\&nrm=iso

SILVA, M.L.B.; DIMENSTEIN, M.D.B. Manejo da crise: encaminhamento e internação psiquiátrica em questão. Arq. bras. psicol. [Internet]. v. 66, n. 3, p. 31-46. 2014. Disponível em: http://pepsic.bvsalud.org/scielo.php?script=sci_arttext\&pid=S1809-

$52672014000300004 \& \operatorname{lng}=$ pt. 
SILVA, M.V. A clínica integral: o paradigma "psicossocial" como uma exigência da clínica das psicoses. Rev. In-Tensa. Ex-Tensa, Salvador, v. 1, n. 1, p. 40-41. 2007.

SILVA, N.G.; SILVA, P.P.; OLIVEIRA, A.G.B. A percepção dos trabalhadores de enfermagem sobre a assistência à saúde mental em hospital universitário. Revista Ciência e Cuidado em Saúde. v. 11, n. 2, p. 303-310. 2012. DOI: http://dx.doi.org/10.4025/cienccuidsaude.v11i2.11181

ZEFERINO, M.T. Crise e Urgência em Saúde Mental: organização da atenção psicossocial à crise em rede de cuidado. $4^{\mathrm{a}}$ Edição - Florianópolis (SC): Universidade Federal de Santa Catarina, 2015. 97 p. ISBN: 978-85-8328-022-4.

\section{Como citar este artigo (Formato ABNT):}

SILVA FILHO, José Adelmo da; BRAGA, Vanessa de Morais; HOLANDA, Maria do Livramento Alencar de. Saberes e Práticas de uma Equipe Multiprofissional: atendimento à crise no Centro de Atenção Psicossocial. Id on Line Rev.Mult. Psic., 2018, vol.12, n.42, p. 862-874. ISSN: 1981-1179.

Recebido: $10 / 06 / 2018$

Aceito: 26/10/2018 